\title{
Cilostazol and Probucol for Cognitive Decline after Stroke: A Cognitive Outcome Substudy of the PICASSO Trial
}

\author{
Jae-Sung Lim, ${ }^{\text {a } S u n ~ U . ~ K w o n, ~}{ }^{\mathrm{b}}$ Kyung-Ho Yu, ${ }^{\mathrm{a}}$ Sungwook Yu, ${ }^{\mathrm{c}}$ Jong-Ho Park, ${ }^{\mathrm{d}}$ Byung-Chul Lee, ${ }^{\mathrm{a}}$ Mi Sun Oh, \\ Yong-Jae Kim, ${ }^{\mathrm{e}}$ Joung-Ho Rha, ${ }^{\mathrm{f}}$ Yang-Ha Hwang, ${ }^{,}$Ji Sung Lee, ${ }^{\mathrm{h}}$ Sung Hyuk Heo, ${ }^{\mathrm{i}}$ Seong Hwan Ahn, ${ }^{\mathrm{j}}$ \\ Woo-Keun Seo, ${ }^{\mathrm{k}}$ Jong-Moo Park, Ju-Hun Lee, ${ }^{\mathrm{m}}$ Jee-Hyun Kwon, ${ }^{\mathrm{n}}$ Sung-Il Sohn, ${ }^{\mathrm{o}}$ Jin-Man Jung, ${ }^{\mathrm{p}}$ \\ Hahn Young Kim, ${ }^{\mathrm{q}}$ Eung-Gyu Kim, ${ }^{\mathrm{r}}$ Jae-Kwan Cha, ${ }^{\mathrm{s}}$ Man-Seok Park, ${ }^{\mathrm{t}}$ Hyo SukNam, ${ }^{\mathrm{u}}$ Hee-Joon Bae, \\ Dong-Eog Kim, ${ }^{\mathrm{w}}$ Jaeseol Park, ${ }^{\mathrm{a}}$ Yeonwook Kang, ${ }^{\mathrm{x}}$ Jimi Choi, ${ }^{\mathrm{y}}$ Juneyoung Lee ${ }^{\mathrm{y}, \mathrm{z}}$ \\ aDepartment of Neurology, Hallym University Sacred Heart Hospital, Hallym Neurological Institute, Hallym University College of Medicine, \\ Anyang, Korea \\ ${ }^{b}$ Department of Neurology, Asan Medical Center, University of Ulsan College of Medicine, Seoul, Korea \\ 'Department of Neurology, Korea University Anam Hospital, Korea University College of Medicine, Seoul, Korea \\ 'Department of Neurology, Myongji Hospital, Hanyang University College of Medicine, Goyang, Korea \\ 'Department of Neurology, Eunpyeong St. Mary's Hospital, College of Medicine, The Catholic University of Korea, Seoul, Korea \\ fDepartment of Neurology, Inha University Hospital, Incheon, Korea \\ ${ }^{9}$ Department of Neurology, Kyungpook National University Hospital, School of Medicine, Kyungpook National University, Daegu, Korea \\ ${ }^{\text {h}}$ Clinical Research Center, Asan Institute for Life Sciences, Asan Medical Center, Seoul, Korea \\ 'Department of Neurology, Kyung Hee University Medical Center, Seoul, Korea \\ 'Department of Neurology, Chosun University Hospital, Gwangju, Korea \\ kDepartment of Neurology, Samsung Medical Center, Sungkyunkwan University School of Medicine, Seoul, Korea \\ 'Department of Neurology, Nowon Eulji Medical Center, Eulji University, Seoul, Korea \\ ${ }^{m}$ Department of Neurology, Hallym University Kangdong Sacred Heart Hospital, Hallym University College of Medicine, Seoul, Korea \\ "Department of Neurology, Ulsan University Hospital, University of Ulsan College of Medicine, Ulsan, Korea \\ 'Department of Neurology, Keimyung University Dongsan Hospital, Keimyung University School of Medicine, Daegu, Korea \\ PDepartment of Neurology, Korea University Ansan Hospital, Ansan, Korea

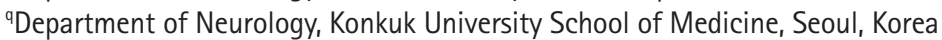 \\ 'Department of Neurology, Inje University Busan Paik Hospital, Inje University College of Medicine, Busan, Korea \\ sDepartment of Neurology, Dong-A University Hospital, Busan, Korea \\ tDepartment of Neurology, Chonnam National University Hospital, Chonnam National University Medical School, Gwangju, Korea \\ "Department of Neurology, Severance Hospital, Yonsei University College of Medicine, Seoul, Korea \\ 'Department of Neurology, Seoul National University Bundang Hospital, Seoul National University College of Medicine, Seongnam, Korea \\ ${ }^{w}$ Department of Neurology, Dongguk University Ilsan Hospital, Goyang, Korea \\ ×Department of Psychology, Hallym University, Chuncheon, Korea \\ 'Department of Biostatistics, Korea University College of Medicine, Seoul, Korea \\ zBK21 FOUR R\&E Center for Learning Health Systems, Korea University, Seoul, Korea
}

\section{Dear Sir:}

Previous clinical trials to prevent post-stroke cognitive impairment, such as Prevention Regimen for Effectively Avoiding Second Strokes (PRoFESS) and Secondary Prevention of Small Subcortical Strokes (SPS3), failed to show clinically meaningful results. ${ }^{1,2}$ There is an evidence that cilostazol, a phosphodies- terase- 3 inhibitor, could suppress cognitive decline in patients with dementia, ${ }^{3}$ and decrease amyloid beta accumulation. ${ }^{4}$ Probucol, a cholesteryl ester transfer protein activator with lipid-lowering and anti-oxidative effects, has a beneficial effect on cognition by inhibiting amyloid beta-induced hippocampal synaptic impairment. ${ }^{5}$ Thus, we aimed to determine the efficacy of cilostazol and probucol for preventing poststroke cogni- 
tive decline in patients with multiple cerebral microbleeds (CMBs) or a history of prior intracerebral hemorrhage (ICH); a population that is expected to have a high risk for future cognitive decline.

Preventlon of CArdiovascular events in iSchemic Stroke patients with high risk of cerebral hemOrrhage for reducing COGnitive decline (PICASSO-COG) is a predetermined substudy of the PICASSO trial, which is a randomized double-blinded placebo-controlled trial with a $2 \times 2$ factorial design: cilostazol versus aspirin, and probucol versus no probucol. ${ }^{6}$ The design and analysis plan have been previously reported. 'The key inclusion criteria were non-cardioembolic ischemic stroke or transient ischemia attack and previous ICH or multiple CMBs on gradient echo imaging. Cognitive function was assessed using the Mini-Mental State Examination (MMSE) and the Mon- treal Cognitive Assessment (MoCA) at randomization and at 4, $13,25,37$, and 49 months after randomization. The cognitive function at the second visit (4 months after randomization) in patients who were randomized within 3 months after stroke was set as the baseline function, while the cognitive function at the first visit ( 1 month after enrollment) was set as the baseline for those randomized beyond 90 days after stroke. The baseline cognitive assessment was therefore conducted between 4 and 7 months after stroke onset in all participants eligible for the PICASSO-COG substudy. The primary outcome was a change in MMSE score, and a restricted maximum likelihoodbased mixed effects model with repeated measurements was used. The efficacy of each treatment was analyzed separately because the interaction effect between the antiplatelets and lipid-lowering treatment was not significant. Detailed informa-
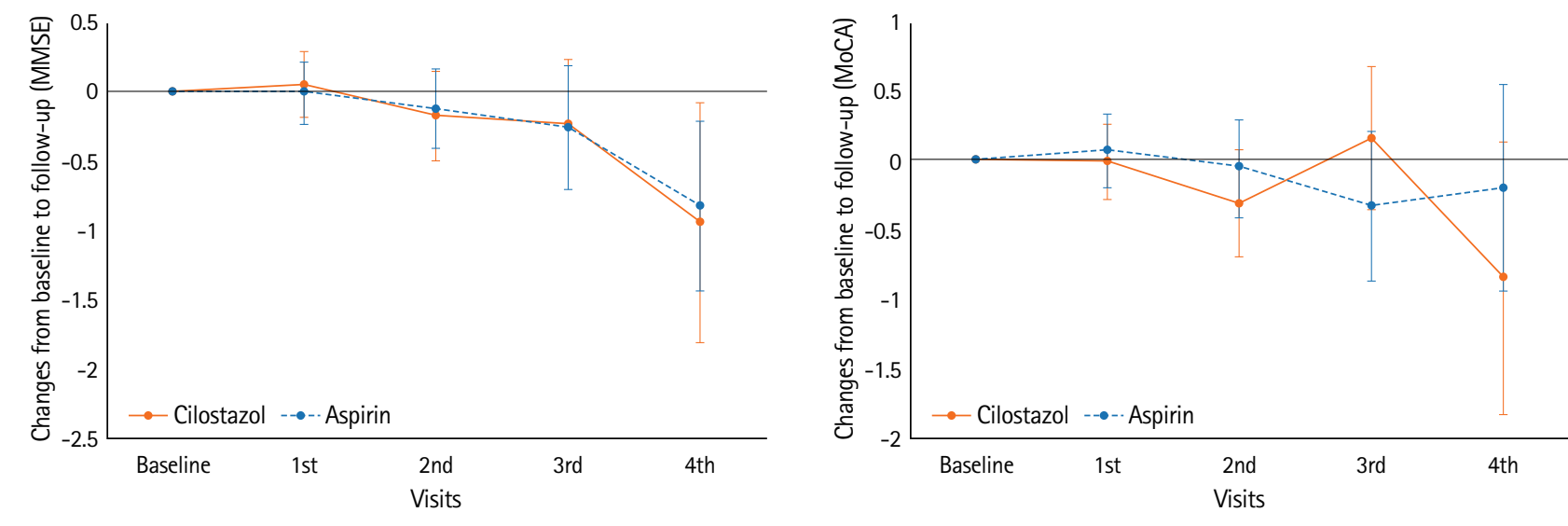

$\begin{array}{rllllll}\text { Cilostazol } & 451 & 449 & 301 & 181 & 66 & \\ \text { Aspirin } & 441 & 439 & 292 & 180 & 72 & \text { A }\end{array}$

$\begin{array}{rllllll}\text { Cilostazol } & 447 & 444 & 297 & 178 & 64 & \\ \text { Aspirin } & 430 & 427 & 285 & 176 & 68 & \text { B }\end{array}$
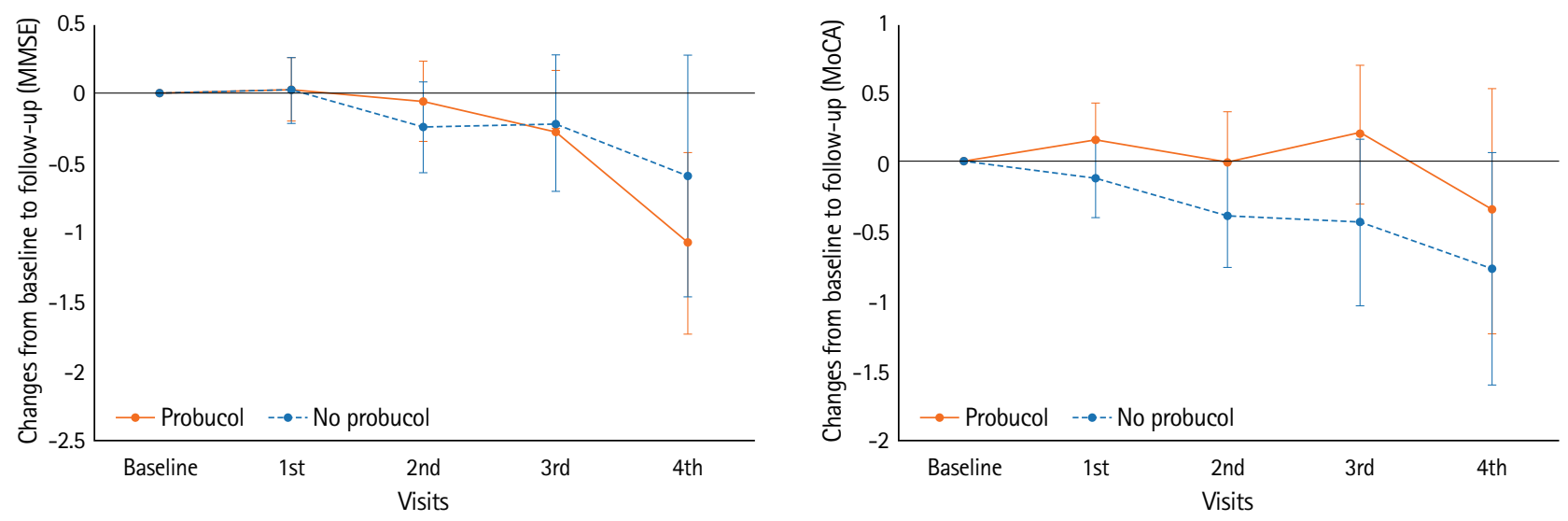

$\begin{array}{rllllll}\text { Probucol } & 459 & 456 & 303 & 197 & 82 & \\ \text { No probucol } & 433 & 432 & 290 & 164 & 56 & \\ & & & & & & \text { C }\end{array}$

$\begin{array}{rllllll}\text { Probucol } & 452 & 447 & 298 & 192 & 77 & \\ \text { No probucol } & 425 & 424 & 284 & 162 & 55 & \\ & & & & & & \end{array}$

Figure 1. Mean changes in cognitive scores from baseline to each follow-up in $(A, B)$ cilostazol vs. aspirin and $(C, D)$ probucol vs. no probucol groups. $(A, C)$ Mini-Mental State Examination (MMSE) and (B, D) Montreal Cognitive Assessment (MoCA). 
tion on the analyses, including sensitivity and subgroup analyses, are presented in the Supplementary methods.

As shown in Supplementary Figure 1, among 1,382 subjects, 1,240 completed cognitive evaluations at randomization and 892 subjects ( 877 for the MoCA) were finally included (Supplementary Table 1).? The baseline characteristics were not significantly different between the treatment groups, except the proportion of those with baseline MMSE $\leq 24$ (Supplementary Table 2). ${ }^{1}$ Cilostazol did not show any significant differences in preventing cognitive decline in comparison with aspirin (Figure 1 and Supplementary Table 3). In the subgroup analysis according to the baseline MMSE score, the decrease in the MMSE score in the aspirin group of those with baseline MMSE $\leq 24$ was more pronounced than that in the cilostazol group although the treatment effect was not significant (Supplementary Table 4). In the propensity score-matched subsets considering the baseline differences in the proportions of those with baseline MMSE $\leq 24$, the cilostazol group showed a favorable outcome in those with mild to moderate white matter hyperintensities (WMHs) (Supplementary Table 5). Otherwise, no significant results were found in the subgroups and sensitivity analysis. Probucol treatment did not show any beneficial effect in the primary outcome using the MMSE. When analyzed according to the MoCA scores, probucol showed a favorable effect in preventing cognitive decline compared with the no probucol group (Supplementary Table 3). This effect was also observed in the subgroups without diabetes mellitus, with concomitant lipid-lowering agents, with baseline MMSE $>24$, and without severe WMH (Supplementary Table 4).

Longitudinal cognitive profiles of the study population might explain why this trial failed to prove the hypothesis. The demographics of the study subjects were comparable to those of the SPS3 trial. ${ }^{2}$ However, $69.3 \%$ of the PICASSO-COG subjects had moderate or severe $\mathrm{WMH}$, while half of the subjects in the SPS3 had none or mild WMH. In this distinctive population, the magnitude of observed cognitive change was smaller than what we had expected. ${ }^{7}$ There are several reasons to consider. It has been reported that cognitive decline in patients with moderate to severe WMH was mainly observed in processing speed and executive function. ${ }^{8}$ In the SPS3 trial conducted in patients with lacunar infarction, verbal fluency was mainly impaired in addition to episodic memory. ${ }^{9}$ Memory dysfunction has also been reported to be affected by actually mediating executive dysfunction. ${ }^{8}$ Therefore, the MMSE was not sensitive enough to capture these long-term cognitive changes. The MoCA has been reported to be more sensitive to the stroke population than the MMSE; however, the MoCA total score seems inadequate to quantify changes over a 2-year study pe- riod. For subsequent clinical trials, neuropsychological tests that more sensitively assess changes over time in the target population, such as fluency, trail-making, and the Stroop test should be adopted. In another aspect, the active risk factor control in the trial setting might prevent the cognitive deterioration of study subjects, including the control group, and made it difficult to verify the effectiveness of the trial drug. This can be conceived from the findings from the previous trials for vascular cognitive impairment, which showed stable cognitive trajectories in placebo arms. ${ }^{10}$ Lastly, it is possible that the heterogeneity of WMH might have been affected." The theoretically hypothesized cognitive decline might not be actually observed in patients with WMH of causes other than ischemic origin. However, since the subjects of this trial had ischemic stroke based on the inclusion criteria and had preceding $\mathrm{ICH} /$ multiple CMBs, the proportion of these patients is not expected to be high.

We predetermined the time window of baseline cognitive evaluation between 4 and 7 months after entry event to minimize the effects of acute stroke on cognitive function. ${ }^{12}$ The intervals between index-stroke and baseline evaluations were 1 month in the PRoFESS trial and 74 to 76 days in the SPS3 trial. ${ }^{1,2}$ If we were to include the spontaneous cognitive recovery after stroke in our analysis, the effects of the study medication could be exaggerated or underestimated.

As a limitation, the current study population did not seem to fulfill the criteria of reliable cognitive decline, and the trial needed much longer follow-up to show a significant change in the MMSE score. ${ }^{7}$ In addition, a treatment effect could have occurred between the index-stroke and the baseline assessment. Since we limited our analysis to those who underwent baseline evaluations for 4 to 7 months after index-stroke, we could not address this possibility in our analysis.

To the best of our knowledge, this is the first clinical trial comparing the efficacy of aspirin, cilostazol, and probucol in preventing poststroke cognitive decline. Cilostazol and probucol did not show any significant differences compared to aspirin and no probucol. However, when patients were assessed by the MoCA, probucol reduced cognitive decline after stroke.

\section{Supplementary materials}

Supplementary materials related to this article can be found online at https://doi.org/10.5853/jos.2020.03650.

\section{References}

1. Diener HC, Sacco RL, Yusuf S, Cotton D, Ounpuu S, Lawton 
WA, et al. Effects of aspirin plus extended-release dipyridamole versus clopidogrel and telmisartan on disability and cognitive function after recurrent stroke in patients with ischaemic stroke in the Prevention Regimen for Effectively Avoiding Second Strokes (PRoFESS) trial: a double-blind, active and placebo-controlled study. Lancet Neurol 2008;7: 875-884.

2. Pearce LA, McClure LA, Anderson DC, Jacova $C$, Sharma $M$, Hart RG, et al. Effects of long-term blood pressure lowering and dual antiplatelet treatment on cognitive function in patients with recent lacunar stroke: a secondary analysis from the SPS3 randomised trial. Lancet Neurol 2014;13:11771185.

3. Ihara $M$, Nishino $M$, Taguchi $A$, Yamamoto $Y$, Hattori $Y$, Saito $S$, et al. Cilostazol add-on therapy in patients with mild dementia receiving donepezil: a retrospective study. PLoS One 2014;9:e89516.

4. Park SH, Kim JH, Bae SS, Hong KW, Lee DS, Leem JY, et al. Protective effect of the phosphodiesterase III inhibitor cilostazol on amyloid $\beta$-induced cognitive deficits associated with decreased amyloid $\beta$ accumulation. Biochem Biophys Res Commun 2011;408:602-608.

5. Santos $D B$, Peres $K C$, Ribeiro $R P$, Colle $D$, dos Santos $A A_{\text {, }}$ Moreira EL, et al. Probucol, a lipid-lowering drug, prevents cognitive and hippocampal synaptic impairments induced by amyloid $\beta$ peptide in mice. Exp Neurol 2012;233:767-775.

6. Kim BJ, Lee EJ, Kwon SU, Park JH, Kim YJ, Hong KS, et al. Prevention of cardiovascular events in Asian patients with ischaemic stroke at high risk of cerebral haemorrhage (PICASSO): a multicentre, randomised controlled trial. Lancet Neurol 2018;17:509-518.

7. Yu KH, Hong KS, Oh MS, Lee J, Lee JS, Kwon SU, et al. Design and rationale for a cognitive outcome substudy in ischemic stroke patients with high risk of cerebral hemorrhage. J Stroke Cerebrovasc Dis 2016;25:2061-2066.

8. Jokinen $H$, Kalska $H$, Mäntylä R, Ylikoski R, Hietanen M, Po-

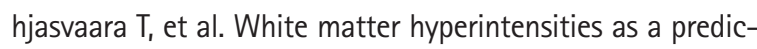
tor of neuropsychological deficits post-stroke. J Neurol Neu- rosurg Psychiatry 2005;76:1229-1233.

9. Jacova C, Pearce LA, Costello R, McClure LA, Holliday SL, Hart $R G$, et al. Cognitive impairment in lacunar strokes: the SPS3 trial. Ann Neurol 2012;72:351-362.

10. Black S, Román GC, Geldmacher DS, Salloway S, Hecker J, Burns $A$, et al. Efficacy and tolerability of donepezil in vascular dementia: positive results of a 24-week, multicenter, international, randomized, placebo-controlled clinical trial. Stroke 2003;34:2323-2330.

11. Pantoni L. Cerebral small vessel disease: from pathogenesis and clinical characteristics to therapeutic challenges. Lancet Neurol 2010;9:689-701.

12. Sachdev PS, Lo JW, Crawford JD, Mellon L, Hickey A, Williams $D$, et al. STROKOG (stroke and cognition consortium): an international consortium to examine the epidemiology, diagnosis, and treatment of neurocognitive disorders in relation to cerebrovascular disease. Alzheimers Dement (Amst) 2016;7:11-23.

Correspondence: Kyung-Ho Yu

Department of Neurology, Hallym University Sacred Heart Hospital, Hallym Neurological Institute, Hallym University College of Medicine, 22 Gwanpyeongro 170beon-gil, Dongan-gu, Anyang 14068, Korea

Tel: +82-31-380-3740

Fax: +82-31-381-9474

E-mail: ykh1030@hallym.or.kr

https://orcid.org/0000-0002-8997-5626

Co-correspondence: Sun U. Kwon

Department of Neurology, Asan Medical Center, University of Ulsan College of Medicine, 88 Olympic-ro 43-gil, Songpa-gu, Seoul 05505, Korea

Tel: +82-2-3010-3960

Fax: +82-2474-4691

E-mail: sukwon@amc.seoul.kr

https://orcid.org/0000-0001-8302-331X

Received: August 31, 2020

Revised: November 14, 2020

Accepted: November 18, 2020

This work was supported by Korea Otsuka Pharmaceutical Company. Sun U. Kwon declares grants from Korea Otsuka Pharmaceutical Company. All other authors declare no competing interests. 


\section{Supplementary methods}

The Preventlon of CArdiovascular events in iSchemic Stroke patients with high risk of cerebral hemOrrhage for reducing COGnitive decline (PICASSO-COG) substudy was conducted only in South Korea (59 centers) because the cognitive assessment tools had not been validated by cross-cultural studies in each language.

The primary outcome was the change in Mini-Mental State Examination (MMSE) score over time from baseline in an intention-to-treat population. A restricted maximum likelihoodbased mixed effects model with repeated measurements (MMRM) was used to compare cognitive changes over time between groups. The model included the fixed categorical effects of treatment group and sex as well as fixed continuous covariates of the patient's age, duration of education, number of visits, baseline cognitive scores, and the National Institutes of Health Stroke Scale (NIHSS) score. The effect of the study sites was adjusted as a random factor in the model. An unstructured covariance structure, common to the treatments, was used to model the within-subject correlation. Although this study had a $2 \times 2$ factorial design, the efficacy of each treatment was analyzed separately because the interaction effect between the antiplatelets and lipid-lowering treatment was not significant.

As cognitive impairment is an independent risk factor for attrition in a longitudinal study, we performed sensitivity analysis to examine its influence on cognitive outcome. Sensitivity analyses included the following: (1) a restricted maximum like- lihood-based MMRM analysis with further adjustment for the participant's drop-out status during the trial period as well as treatment status at the previous visit, which is defined as the patient missing the cognitive evaluation in the scheduled visit before the current visit, in the model; (2) MMRM analyses of participants' cognitive evaluation at baseline and follow-up visits at 13,25,37, and 49 months; (3) MMRM analyses of participants who completed all scheduled visits during the following periods: baseline to 13 months, baseline to 25 months, baseline to 37 months, and baseline to 49 months; and (4) MMRM analyses of participants with ischemic stroke as an entry event excluding transient ischemic attack.

The primary outcome between the comparative arms was compared for the following subgroups: diabetes versus non-diabetes, mild to moderate (Fazekas grade 0-2) versus severe (Fazekas grade 3) white matter hyperintensities on magnetic resonance imaging, baseline MMSE $\leq 24$ versus $>24$, and concomitant use of statin versus non-use. The same analyses as performed in the sensitivity and subgroup analyses were reconducted in the propensity score matched subsets, which were constructed using the variables of subject age, sex, educational years, baseline cognitive score, and baseline NIHSS score to overcome the differences between the treatment groups arising from non-random missing in this substudy.

The longitudinal change in MoCA was also evaluated using the same statistical methods. A two-sided $P$-value of 0.05 was used to indicate statistical significance. All statistical analyses were performed using SAS version 9.3 (SAS Institute, Cary, NC, USA). 
Supplementary Table 1. Baseline characteristics of the included and excluded subjects

\begin{tabular}{|c|c|c|c|c|c|c|c|c|c|}
\hline \multirow[b]{2}{*}{ Characteristic } & \multicolumn{3}{|c|}{ PICASSO-COG study } & \multicolumn{3}{|c|}{ MMSE analysis } & \multicolumn{3}{|c|}{ MoCA analysis } \\
\hline & $\begin{array}{l}\text { Included } \\
(n=1,240)\end{array}$ & $\begin{array}{c}\text { Excluded } \\
(n=142)\end{array}$ & $P$ & $\begin{array}{l}\text { Included } \\
(n=892)\end{array}$ & $\begin{array}{l}\text { Excluded } \\
(n=348)\end{array}$ & $P$ & $\begin{array}{l}\text { Included } \\
(\mathrm{n}=877)\end{array}$ & $\begin{array}{l}\text { Excluded } \\
(n=363)\end{array}$ & $P$ \\
\hline Age (yr) & $65.8 \pm 10.8$ & $68.3 \pm 10.0$ & 0.01 & $64.9 \pm 10.8$ & $68.2 \pm 10.5$ & $<0.01$ & $64.8 \pm 10.8$ & $68.3 \pm 10.4$ & $<0.01$ \\
\hline Female sex & $480(38.7)$ & $53(37.3)$ & 0.75 & $327(36.7)$ & $153(44.0)$ & 0.02 & $318(36.3)$ & $162(44.6)$ & 0.01 \\
\hline Education (yr) & $9(6-12)$ & $9(6-12)$ & 0.41 & $9(6-12)$ & $6(5-12)$ & $<0.01$ & $9(6-12)$ & $6(5-12)$ & $<0.01$ \\
\hline Hypertension & 1,091 (88.0) & $133(93.7)$ & 0.04 & 796 (89.2) & $295(84.8)$ & 0.03 & 783 (89.3) & $308(84.9)$ & 0.03 \\
\hline Diabetes & $389(31.4)$ & $53(37.3)$ & 0.15 & $276(30.9)$ & $113(32.5)$ & 0.60 & $271(30.9)$ & $118(32.5)$ & 0.58 \\
\hline Hyperlipidemia & $511(41.2)$ & $54(38.0)$ & 0.47 & $373(41.8)$ & $138(40.0)$ & 0.49 & $369(42.1)$ & $142(39.1)$ & 0.34 \\
\hline Use of lipid-lowering agent ${ }^{*}$ & 969 (78.2) & 85 (59.9) & $<0.01$ & 695 (77.9) & $274(78.7)$ & 0.75 & $686(78.2)$ & $283(78.0)$ & 0.92 \\
\hline Coronary artery disease & $59(4.8)$ & $8(5.6)$ & 0.65 & $37(4.2)$ & $22(6.3)$ & 0.11 & $37(4.2)$ & $22(6.1)$ & 0.17 \\
\hline Smoking & $546(44.0)$ & 65 (45.8) & 0.69 & $407(45.6)$ & 139 (39.9) & 0.07 & $404(46.1)$ & $142(39.1)$ & 0.02 \\
\hline Index event & & & 0.01 & & & 0.18 & & & 0.05 \\
\hline Ischemic stroke & $1,175(94.8)$ & $142(100.0)$ & & 850 (95.3) & $325(93.4)$ & & $838(95.6)$ & 337 (92.8) & \\
\hline Transient ischemic attack & $65(5.2)$ & $0(0.0)$ & & $42(4.7)$ & $23(6.6)$ & & $39(4.5)$ & $26(7.2)$ & \\
\hline Baseline NIHSS & $1(0-3)$ & $3(1-5)$ & $<0.01$ & $1(0-3)$ & $2(1-4)$ & $<0.01$ & $1(0-3)$ & $2(1-4)$ & $<0.01$ \\
\hline Baseline MMSE & $26(21-28)$ & - & - & $26(23-29)$ & $24(17-27)$ & $<0.01$ & $26(23-29)$ & $24(17-27)$ & $<0.01$ \\
\hline 24 or less & $492(39.7)$ & - & & $313(35.1)$ & $179(51.4)$ & $<0.01$ & $303(34.6)$ & $189(52.1)$ & $<0.01$ \\
\hline$>24$ & $748(60.3)$ & - & & $579(64.9)$ & $169(48.6)$ & & $574(65.4)$ & $174(47.9)$ & \\
\hline Baseline MoCA & $20(14-24)$ & - & - & $20(16-24)$ & $17(10-22)$ & $<0.01$ & $20(16-24)$ & $17(10-22)$ & $<0.01$ \\
\hline \multicolumn{10}{|l|}{ Treatment } \\
\hline Cilostazol & $618(49.8)$ & $71(50.0)$ & 0.97 & $451(50.6)$ & $167(48.0)$ & 0.42 & $447(51.0)$ & $171(47.1)$ & 0.22 \\
\hline Probucol & $622(50.2)$ & $69(48.6)$ & 0.72 & $459(51.5)$ & $163(46.8)$ & 0.14 & $452(51.5)$ & $170(46.8)$ & 0.13 \\
\hline $\mathrm{SBP}(\mathrm{mm} \mathrm{Hg})$ & $135.4 \pm 18.4$ & $133.7 \pm 19.1$ & 0.30 & $135.4 \pm 18.6$ & $135.5 \pm 17.8$ & 0.96 & $135.3 \pm 18.7$ & $135.6 \pm 17.6$ & 0.79 \\
\hline $\mathrm{DBP}(\mathrm{mm} \mathrm{Hg})$ & $80.1 \pm 11.8$ & $80.9 \pm 12.2$ & 0.43 & $80.1 \pm 11.8$ & $80.2 \pm 11.8$ & 0.88 & $80.1 \pm 11.9$ & $80.2 \pm 11.6$ & 0.85 \\
\hline $\mathrm{BP}$ readings & $7(4-13)$ & $10(3-18)$ & 0.01 & $9(6-13)$ & $2(1-4)$ & $<0.01$ & $9(6-13)$ & $2(1-4)$ & $<0.01$ \\
\hline Follow-up periods (yr) & $1.9(1.0-3.0)$ & $2.7(0.6-4.5)$ & $<0.01$ & $2.1(1.3-3.0)$ & $0.5(0.1-1.1)$ & $<0.01$ & $2.1(1.3-3.0)$ & $0.6(0.1-1.1)$ & $<0.01$ \\
\hline Severe WMH & $324(27.1)$ & $30(22.1)$ & 0.21 & $210(24.5)$ & $114(33.5)$ & $<0.01$ & $206(24.4)$ & $118(33.2)$ & $<0.01$ \\
\hline \multicolumn{10}{|l|}{ Outcome events } \\
\hline Recurrent stroke ${ }^{+}$ & $102(8.2)$ & $9(6.3)$ & 0.43 & $44(4.9)$ & $58(16.7)$ & $<0.01$ & $41(4.7)$ & 61 (16.8) & $<0.01$ \\
\hline Ischemic & $80(6.5)$ & $8(5.6)$ & 0.71 & $31(3.5)$ & $49(14.1)$ & $<0.01$ & $30(3.4)$ & $50(13.8)$ & $<0.01$ \\
\hline Hemorrhagic & $23(1.9)$ & $1(0.7)$ & 0.50 & $14(1.6)$ & $9(2.6)$ & 0.23 & $12(1.4)$ & $11(3.0)$ & 0.048 \\
\hline Myocardial infarction & $8(0.7)$ & $1(0.7)$ & 0.99 & $3(0.3)$ & $5(1.4)$ & 0.04 & $3(0.3)$ & $5(1.4)$ & 0.04 \\
\hline Death & $39(3.2)$ & $9(6.3)$ & 0.08 & $17(1.9)$ & $22(6.3)$ & $<0.01$ & $17(1.9)$ & $22(6.1)$ & $<0.01$ \\
\hline
\end{tabular}

Values are presented as mean \pm standard deviation, number $(\%)$, or median (interquartile range). Severe white matter hyperintensities were defined as Fazekas grade 3.

PICASSO-COG, Preventlon of CArdiovascular events in iSchemic Stroke patients with high risk of cerebral hemOrrhage for reducing COGnitive decline; MMSE, Mini-Mental State Examination; MoCA, Montreal Cognitive Assessment; NIHSS, National Institutes of Health Stroke Scale; SBP, systolic blood pressure; DBP, diastolic blood pressure; BP, blood pressure; $\mathrm{WMH}$, white matter hyperintensity.

*Prior to randomization; ${ }^{+}$One subject had both ischemic and hemorrhagic stroke and was counted as a duplicate. 
Supplementary Table 2. Baseline characteristics of the study subjects

\begin{tabular}{|c|c|c|c|c|}
\hline \multirow{2}{*}{ Characteristic } & \multicolumn{2}{|c|}{ Antiplatelet treatment } & \multicolumn{2}{|c|}{ Lipid-lowering treatment } \\
\hline & Cilostazol $(n=451)$ & Aspirin $(n=441)$ & Probucol $(n=459)$ & No probucol $(n=433)$ \\
\hline Age (yr) & $65.0 \pm 10.8$ & $64.8 \pm 10.8$ & $64.7 \pm 10.8$ & $65.2 \pm 10.8$ \\
\hline Male sex & $283(62.7)$ & $282(63.9)$ & $290(63.2)$ & $275(63.5)$ \\
\hline Education (yr) & $9(6-12)$ & $9(6-12)$ & $9(6-12)$ & $9(6-12)$ \\
\hline \multicolumn{5}{|l|}{ Entry event } \\
\hline Ischemic stroke & $432(95.8)$ & $418(94.8)$ & $441(96.1)$ & $409(94.5)$ \\
\hline Transient ischemic attack & $19(4.2)$ & $23(5.2)$ & $18(3.9)$ & $24(5.5)$ \\
\hline \multicolumn{5}{|l|}{ Index of high risk of ICH } \\
\hline Prior history of $\mathrm{ICH}$ & $69(15.3)$ & $76(17.2)$ & $72(15.7)$ & $73(16.9)$ \\
\hline Imaging findings of ICH without clinical history & $83(18.4)$ & $77(17.5)$ & $87(18.9)$ & $73(16.9)$ \\
\hline Multiple microbleeds ( $\geq 2)$ & $299(66.3)$ & $288(65.3)$ & $300(65.4)$ & $287(66.3)$ \\
\hline Time-to-randomization since entry event (day) & $18(9-41)$ & $18(9-42)$ & $18(9-43)$ & $18(8-41)$ \\
\hline$\leq 10$ & $130(28.8)$ & $132(29.9)$ & $128(27.9)$ & $134(30.9)$ \\
\hline $11-30$ & $177(39.2)$ & $173(39.2)$ & $180(39.2)$ & $170(39.3)$ \\
\hline $31-90$ & $93(20.6)$ & $93(21.1)$ & $95(20.7)$ & $91(21.0)$ \\
\hline$>90$ & $51(11.3)$ & $43(9.8)$ & $56(12.2)$ & $38(8.8)$ \\
\hline Baseline NIHSS & $1(0-3)$ & $1(0-3)$ & $1(0-3)$ & $1(0-3)$ \\
\hline Baseline MMSE & $26(22-28)$ & $27(23-29)$ & $26(22-28)$ & $26(23-29)$ \\
\hline Baseline MMSE $\leq 24$ & $173(38.4)$ & $140(31.7)$ & $160(34.9)$ & $153(35.3)$ \\
\hline Baseline MoCA & $20(15-24)$ & $21(16-24)$ & $20(15.5-24)$ & $21(16-24)$ \\
\hline Time-to-baseline MMSE since entry event (day) & $136(125-148)$ & $135(127-151)$ & $136(126-151)$ & $135(127-148.5)$ \\
\hline \multicolumn{5}{|l|}{ Risk factors } \\
\hline Hypertension & $402(89.1)$ & $394(89.3)$ & $411(89.5)$ & $385(88.9)$ \\
\hline Diabetes mellitus & $134(29.7)$ & $142(32.2)$ & $136(29.6)$ & $140(32.3)$ \\
\hline Dyslipidemia & $183(40.6)$ & $190(43.1)$ & $206(44.9)$ & $167(38.6)$ \\
\hline Current smoking & $93(20.6)$ & $102(23.1)$ & $103(22.4)$ & $92(21.2)$ \\
\hline Coronary artery disease & $15(3.3)$ & $22(5.0)$ & $22(4.8)$ & $15(3.5)$ \\
\hline \multicolumn{5}{|l|}{ Lipids (mg/dL) } \\
\hline Total cholesterol & $165.7 \pm 39.2$ & $169.0 \pm 41.1$ & $170.5 \pm 40.8$ & $164.0 \pm 39.3$ \\
\hline LDL-C & $101.0 \pm 36.0$ & $102.7 \pm 35.4$ & $104.7 \pm 36.4$ & $98.7 \pm 34.7$ \\
\hline HDL-C & $45.2 \pm 11.7$ & $45.9 \pm 12.1$ & $45.5 \pm 12.1$ & $45.5 \pm 11.7$ \\
\hline \multicolumn{5}{|l|}{ Fazekas score for WMH } \\
\hline 0 & $0(0.0)$ & $0(0.0)$ & $0(0.0)$ & $0(0.0)$ \\
\hline 1 & $122(27.1)$ & $141(32.0)$ & $130(29.1)$ & $133(32.4)$ \\
\hline 2 & $194(43.0)$ & $191(43.3)$ & $213(47.6)$ & $172(41.8)$ \\
\hline 3 & $112(24.8)$ & $98(22.2)$ & $104(23.3)$ & $106(25.8)$ \\
\hline \multicolumn{5}{|l|}{ Concomitant therapy } \\
\hline Aspirin (after randomization) & & & $218(47.5)$ & $223(51.5)$ \\
\hline Cilostazol (after randomization) & & & $241(52.5)$ & $210(48.5)$ \\
\hline Probucol & $241(53.4)$ & $218(49.4)$ & & \\
\hline Other lipid-lowering agents & $355(79.1)$ & $353(80.2)$ & $360(78.8)$ & $348(80.6)$ \\
\hline
\end{tabular}

Values are presented as mean \pm standard deviation, number (\%), or median (interquartile range).

ICH, intracerebral hemorrhage; NIHSS, National Institutes of Health Stroke Scale; MMSE, Mini-Mental State Examination; MoCA, Montreal Cognitive Assessment; LDL-C, low-density lipoprotein cholesterol; HDL-C, high-density lipoprotein cholesterol; WMH, white matter hyperintensity. 
Supplementary Table 3. MMRM analysis in cilostazol vs. aspirin and probucol vs. no probucol groups

\begin{tabular}{|c|c|c|c|c|c|c|c|c|c|c|c|c|}
\hline \multirow{3}{*}{ Time point } & \multicolumn{6}{|c|}{ Cilostazol vs. aspirin } & \multicolumn{6}{|c|}{ Probucol vs. no probucol } \\
\hline & \multicolumn{3}{|c|}{ MMSE } & \multicolumn{3}{|c|}{ MoCA } & \multicolumn{3}{|c|}{ MMSE } & \multicolumn{3}{|c|}{ MoCA } \\
\hline & $\begin{array}{c}\text { Cilostazol } \\
(n=451)\end{array}$ & $\begin{array}{c}\text { Aspirin } \\
(n=441)\end{array}$ & $P$ & $\begin{array}{c}\text { Cilostazol } \\
(n=447)\end{array}$ & $\begin{array}{c}\text { Aspirin } \\
(n=430)\end{array}$ & $P$ & $\begin{array}{l}\text { Probucol } \\
(n=459)\end{array}$ & $\begin{array}{l}\text { No probucol } \\
\quad(n=433)\end{array}$ & $P$ & $\begin{array}{l}\text { Probucol } \\
(n=452)\end{array}$ & $\begin{array}{l}\text { No probucol } \\
(n=425)\end{array}$ & $P$ \\
\hline Baseline & $24.76 \pm 4.48$ & $25.07 \pm 4.89$ & $0.81^{*}$ & $18.98 \pm 6.16$ & $19.67 \pm 6.31$ & $0.31^{*}$ & $24.93 \pm 4.73$ & $24.90 \pm 4.65$ & $0.57^{*}$ & $19.23 \pm 6.15$ & $19.42 \pm 6.33$ & $0.01^{*}$ \\
\hline 1st Follow-up & $24.85 \pm 4.65$ & $25.10 \pm 4.95$ & $<0.01^{+}$ & $19.02 \pm 6.37$ & $19.78 \pm 6.62$ & $0.045^{+}$ & $25.00 \pm 5.03$ & $24.94 \pm 4.55$ & $<0.01^{+}$ & $19.44 \pm 6.43$ & $19.34 \pm 6.58$ & $0.03^{+}$ \\
\hline 2nd Follow-up & $24.61 \pm 5.12$ & $25.13 \pm 4.92$ & & $18.75 \pm 6.66$ & $19.91 \pm 6.57$ & & $24.91 \pm 5.03$ & $24.83 \pm 5.03$ & & $19.31 \pm 6.81$ & $19.33 \pm 6.46$ & \\
\hline 3rd Follow-up & $24.56 \pm 5.29$ & $24.82 \pm 5.35$ & & $19.01 \pm 6.80$ & $19.43 \pm 6.96$ & & $24.81 \pm 5.17$ & $24.54 \pm 5.48$ & & $19.67 \pm 6.72$ & $18.69 \pm 7.04$ & \\
\hline 4th Follow-up & $23.97 \pm 5.51$ & $25.15 \pm 5.41$ & & $18.69 \pm 7.34$ & $20.62 \pm 6.98$ & & $24.41 \pm 5.69$ & $24.84 \pm 5.17$ & & $19.91 \pm 7.48$ & $19.36 \pm 6.82$ & \\
\hline
\end{tabular}

Values are presented as mean \pm standard deviation. $P$-values for treatment-by-time interaction were not significant in any analysis.

MMRM, maximum likelihood-based mixed effects model with repeated measurements; MMSE, Mini-Mental State Examination; MoCA, Montreal Cognitive Assessment.

${ }^{*} P$-value for MMRM for treatment effect; ${ }^{+} P$-value for MMRM for the time effect. 
Supplementary Table 4. Subgroup analysis of the cilosatzol/aspirin groups and probucol/no probucol groups

\begin{tabular}{|c|c|c|c|c|c|}
\hline Variable & Baseline & 1st Follow-up & 2nd Follow-up & 3rd Follow-up & 4th Follow-up \\
\hline \multicolumn{6}{|c|}{ Cilosatzol/aspirin MMSE scores } \\
\hline \multicolumn{6}{|c|}{ Baseline MMSE $\leq 24(n=313)$} \\
\hline Cilostazol $(n=173)$ & $20.10 \pm 3.56$ & $20.70 \pm 4.52$ & $19.84 \pm 5.03$ & $19.94 \pm 5.26$ & $19.40 \pm 5.21$ \\
\hline Aspirin $(n=140)$ & $19.23 \pm 4.41$ & $19.73 \pm 5.10$ & $20.01 \pm 5.44$ & $19.67 \pm 5.90$ & $16.54 \pm 6.15$ \\
\hline$P$ & $0.83^{*}$ & $0.01^{+}$ & & & \\
\hline \multicolumn{6}{|c|}{ Baseline MMSE >24 $(n=579)$} \\
\hline Cilostazol $(n=278)$ & $27.66 \pm 1.65$ & $27.41 \pm 2.30$ & $27.28 \pm 2.62$ & $27.40 \pm 2.64$ & $26.76 \pm 3.46$ \\
\hline Aspirin $(n=301)$ & $27.79 \pm 1.65$ & $27.56 \pm 2.14$ & $27.34 \pm 2.39$ & $27.26 \pm 2.67$ & $27.26 \pm 2.16$ \\
\hline$P$ & $0.82^{*}$ & $0.01^{+}$ & & & \\
\hline \multicolumn{6}{|c|}{ Probucol/no probucol MoCA scores } \\
\hline \multicolumn{6}{|l|}{ Diabetes mellitus $(n=271)$} \\
\hline Probucol $(n=135)$ & $18.70 \pm 6.29$ & $18.68 \pm 6.61$ & $18.17 \pm 6.80$ & $18.16 \pm 6.85$ & $18.59 \pm 8.46$ \\
\hline No probucol $(n=136)$ & $18.82 \pm 6.06$ & $18.71 \pm 6.14$ & $18.52 \pm 6.00$ & $17.72 \pm 6.28$ & $18.05 \pm 6.76$ \\
\hline$P$ & $0.16^{*}$ & $0.18^{+}$ & & & \\
\hline \multicolumn{6}{|c|}{ No diabetes mellitus $(n=606)$} \\
\hline Probucol $(n=317)$ & $19.45 \pm 6.09$ & $19.77 \pm 6.34$ & $19.77 \pm 6.78$ & $20.19 \pm 6.61$ & $20.28 \pm 7.22$ \\
\hline No probucol $(n=289)$ & $19.70 \pm 6.45$ & $19.63 \pm 6.77$ & $19.72 \pm 6.65$ & $19.21 \pm 7.40$ & $20.24 \pm 6.82$ \\
\hline$P$ & $0.02^{*}$ & $0.01^{+}$ & & & \\
\hline \multicolumn{6}{|c|}{ Concomitant lipid-lowering agents ( $\mathrm{n}=699$ ) } \\
\hline Probucol $(n=355)$ & $19.46 \pm 6.08$ & $19.73 \pm 6.28$ & $19.81 \pm 6.60$ & $20.06 \pm 6.51$ & $20.69 \pm 7.19$ \\
\hline No probucol $(n=344)$ & $19.47 \pm 6.35$ & $19.44 \pm 6.62$ & $19.59 \pm 6.38$ & $19.23 \pm 6.70$ & $19.45 \pm 6.73$ \\
\hline$P$ & $0.08^{*}$ & $0.38^{+}$ & & & \\
\hline \multicolumn{6}{|c|}{ No concomitant lipid-lowering agents $(n=175)$} \\
\hline Probucol $(n=95)$ & $18.35 \pm 6.41$ & $18.44 \pm 6.94$ & $17.71 \pm 7.30$ & $18.58 \pm 7.28$ & $17.06 \pm 8.23$ \\
\hline No probucol $(n=80)$ & $19.21 \pm 6.34$ & $18.84 \pm 6.46$ & $18.30 \pm 6.75$ & $16.62 \pm 7.95$ & $19.13 \pm 7.30$ \\
\hline$P$ & $0.03^{*}$ & $0.03^{+}$ & & & \\
\hline \multicolumn{6}{|c|}{ Baseline MMSE $\leq 24(n=303)$} \\
\hline Probucol $(n=155)$ & $12.94 \pm 4.90$ & $12.90 \pm 5.11$ & $12.60 \pm 5.79$ & $13.21 \pm 5.82$ & $11.92 \pm 6.41$ \\
\hline No probucol $(n=148)$ & $12.86 \pm 5.10$ & $12.78 \pm 5.13$ & $12.17 \pm 4.89$ & $11.61 \pm 5.64$ & $8.55 \pm 5.43$ \\
\hline$P$ & $0.18^{*}$ & $<0.01^{+}$ & & & \\
\hline \multicolumn{6}{|c|}{ Baseline MMSE >24 $(n=574)$} \\
\hline Probucol $(n=297)$ & $22.51 \pm 3.69$ & $22.78 \pm 4.03$ & $22.75 \pm 4.26$ & $23.14 \pm 4.10$ & $23.75 \pm 4.22$ \\
\hline No probucol $(n=277)$ & $22.92 \pm 3.52$ & $22.82 \pm 4.17$ & $22.55 \pm 4.05$ & $22.42 \pm 4.30$ & $22.07 \pm 3.77$ \\
\hline$P$ & $0.01^{*}$ & $0.88^{+}$ & & & \\
\hline \multicolumn{6}{|c|}{ Mild to moderate white matter hyperintensities ( $n=637$ ) } \\
\hline Probucol $(n=338)$ & $20.37 \pm 5.51$ & $20.75 \pm 5.86$ & $20.70 \pm 6.10$ & $20.97 \pm 5.95$ & $21.42 \pm 6.42$ \\
\hline No probucol $(n=299)$ & $20.48 \pm 5.91$ & $20.43 \pm 6.15$ & $20.47 \pm 5.76$ & $20.16 \pm 6.10$ & $20.85 \pm 5.28$ \\
\hline$P$ & $<0.01^{*}$ & $0.40^{+}$ & & & \\
\hline \multicolumn{6}{|c|}{ Severe white matter hyperintensities $(n=206)$} \\
\hline Probucol $(n=102)$ & $15.49 \pm 6.58$ & $15.18 \pm 6.44$ & $14.69 \pm 7.06$ & $15.35 \pm 7.41$ & $14.20 \pm 8.85$ \\
\hline No probucol $(n=104)$ & $16.35 \pm 6.61$ & $16.03 \pm 6.82$ & $15.99 \pm 7.24$ & $14.05 \pm 7.87$ & $15.30 \pm 9.29$ \\
\hline$P$ & $0.79^{*}$ & $0.01^{+}$ & & & \\
\hline
\end{tabular}

Values are presented as mean \pm standard deviation. Mild to moderate white matter hyperintensities were defined as Fazekas grade 1 or 2 , and severe white matter hyperintensities as Fazekas grade 3. $P$-values for treatment by time interactions were not significant for any analysis.

MMSE, Mini-Mental State Examination; MoCA, Montreal Cognitive Assessment.

${ }^{*} P$-value by MMRM for the treatment effect; ${ }^{+} P$-value by MMRM for the time effect. 
Supplementary Table 5. Comparisons of MMSE scores between cilostazol and aspirin group according to severity of white matter changes in propensity score matched subsets

\begin{tabular}{lccccccc}
\hline \multirow{2}{*}{ MMSE scores } & \multicolumn{2}{c}{ Mild to moderate white matter hyperintensities $(n=574)$} & & \multicolumn{3}{c}{ Severe white matter hyperintensities $(n=148)$} \\
\cline { 2 - 3 } & Cilostazol $(n=287)$ & Aspirin $(n=287)$ & $P$ & & Cilostazol $(n=74)$ & Aspirin $(n=74)$ & $P$ \\
\hline Baseline & $25.85 \pm 3.79$ & $25.85 \pm 4.25$ & $0.02^{*}$ & & $22.66 \pm 4.69$ & $22.27 \pm 6.01$ & $0.12^{*}$ \\
1st Follow-up & $26.14 \pm 3.59$ & $25.99 \pm 4.36$ & $0.26^{+}$ & & $21.95 \pm 5.36$ & $22.01 \pm 5.72$ & $<0.01^{+}$ \\
2nd Follow-up & $26.08 \pm 3.99$ & $25.83 \pm 4.05$ & & & $21.79 \pm 5.85$ & $21.74 \pm 6.81$ \\
3rd Follow-up & $26.43 \pm 3.30$ & $25.47 \pm 4.63$ & & $20.03 \pm 6.53$ & $21.47 \pm 7.16$ \\
4th Follow-up & $26.30 \pm 3.06$ & $25.75 \pm 5.18$ & & $16.90 \pm 6.87$ & $20.73 \pm 7.04$ \\
\hline
\end{tabular}

Values are presented as mean \pm standard deviation. The propensity score was calculated using variables, including the participant's age, sex, duration of education, baseline National Institutes of Health Stroke Scale (NIHSS) score, baseline MMSE score, baseline Montreal Cognitive Assessment (MoCA) score, coronary artery disease (yes/no), hypertension (yes/no), systolic blood pressure, and pattern of measurement within each white matter hyperintensity. Mild to moderate white matter hyperintensities were defined as Fazekas grade 1 or 2, and severe white matter hyperintensities as Fazekas grade 3. $P$-values for treatment by time interactions were not significant for any analysis.

MMSE, Mini-Mental State Examination.

${ }^{*} P$-value by MMRM for the treatment effect; ${ }^{+} P$-value by MMRM for the time effect. 


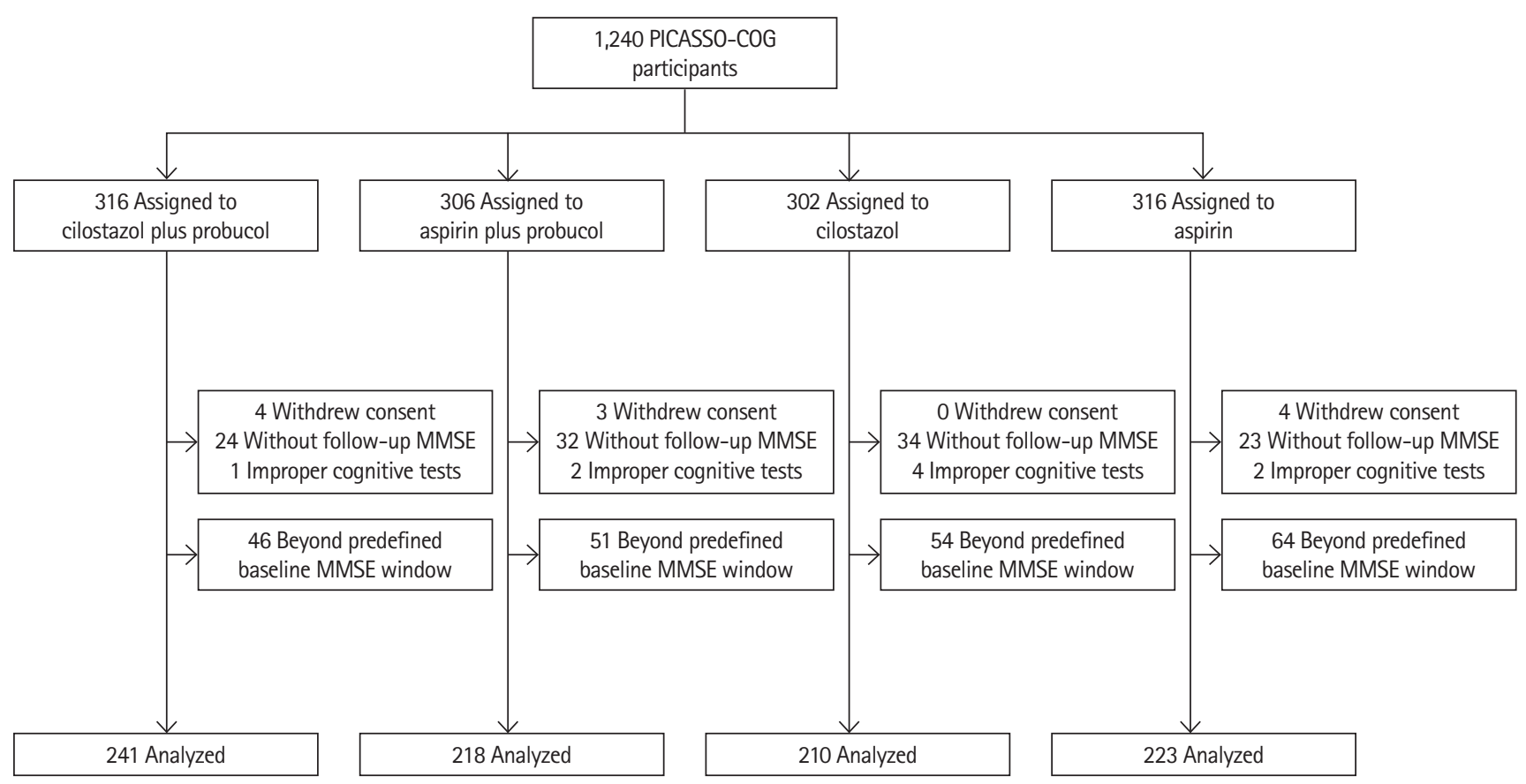

Supplementary Figure 1. Flow diagram of subject enrollment. PICASSO-COG, Preventlon of CArdiovascular events in iSchemic Stroke patients with high risk of cerebral hemOrrhage for reducing COGnitive decline; MMSE, Mini-Mental State Examination. 